\title{
Contribución al conocimiento de los cangrejos (Meiura: Decapoda) de la Colección CEBUC, con registros adicionales en Colombia
}

\author{
Contribution to the knowledge of the crabs (Meiura: Decapoda) of the CEBUC Collection, with \\ additional records in Colombia
}

\author{
Juan Mateo Rivera-Pérez ${ }^{1 *}$, Camilo Andrés Llano-Arias², Lucimar Gomes-Días²
}

\begin{abstract}
Resumen
Las colecciones biológicas constituyen un registro de la historia natural de las diferentes regiones y brindan información relevante para diversas investigaciones científicas concernientes con sistemática, biogeografía, y biología de la conservación, entre otras. En las colecciones relacionadas con artrópodos de Colombia, los cangrejos (Decapoda: Anomura y Brachyura) están poco representados, a pesar de ser el segundo país con la mayor diversidad de cangrejos de agua dulce a nivel mundial y poseer numerosos representantes de especies marinas y semiterrestres, tanto del Atlántico como del Pacífico. La pequeña representatividad del grupo en colecciones biológicas puede deberse al número reducido de especialistas en el grupo y al número limitado de muestreos. El objetivo de este trabajo fue ampliar el conocimiento de los decápodos de Colombia mediante la revisión de los especímenes depositados en la colección CEBUC. Se encontraron 98 individuos distribuidos en 15 familias, 23 géneros y 22 especies. Los ejemplares revisados corresponden a la región Centro Occidental y costa atlántica de Colombia, en donde Caldas es el departamento mejor representado, con el $50 \%$ de los registros. En este trabajo se registran ampliaciones altitudinales para la distribución de Hypolobocera bouvieri y Strengeriana fuhrmanni (Pseudothelphusidae). Además, se documentan ampliaciones en el rango de distribución para otras especies. Este trabajo constituye una fuente preliminar de información sobre la diversidad de los cangrejos a nivel regional y enfatiza que la diversidad puede incrementarse substancialmente con la ampliación del número de investigaciones y la revisión de colecciones biológicas.
\end{abstract}

Palabras claves: Anomura, Brachyura, colecciones biológicas, diversidad, sistematización

\begin{abstract}
Biological collections constitute a record of the natural history of different regions and provide relevant information for various scientific investigations related to systematics, biogeography, conservation biology, among others. In collections associated to arthropods in Colombia, crabs (Meiura) formed by the infraorders Anomura and Brachyura (Decapoda) are poorly represented, despite being the second country with the most diversity of freshwater crabs worldwide and having many representatives of marine and semi-terrestrial species from the Atlantic and Pacific. The small representativeness of the group in the collections could be due to the small number of specialist researchers in the group and the small amount of samplings. The objective of this work was to expand the knowledge on decapods from Colombia through the review of the specimens deposited in the CEBUC collection. We found 98 individuals distributed in 15 families, 23 genera and 22 species. The material reviewed comes from the Central Western and Atlantic coastal region of Colombia, where Caldas is the best represented department, with $50 \%$ of the records. In this work altitudinal enlarge-
\end{abstract}

\footnotetext{
1. Universidade Federal do Pará/EMBRAPA, Pará, Brasil.

2. Universidad de Caldas, Manizales, Colombia.

* Autor para correspondencia: <jumaripe123@gmail.com>
} 
ments are registered for the distribution of Hypolobocera bouvieri and Strengeriana fuhrmanni (Pseudothelphusidae). In addition, expansions in the range of distribution are documented for other species. This work constitutes a preliminary source of information on the diversity of crabs (Meiura) at the regional level and reflects that diversity can increase substantially with the expansion of the number of investigations and the review of biological collections.

Keywords: Anomura, Brachyura, Biological collections, diversity, systematization

\section{INTRODUCCIÓN}

En Colombia, el orden Decapoda se encuentra representado por 1014 especies, aproximadamente (SIB Colombia, 2021). El grupo está compuesto principalmente por camarones, langostas y cangrejos, los cuales son considerados fundamentales en la dinámica de los ecosistemas (Ardila et al., 2002; Cumberlidge et al., 2009). Entre las funciones ecológicas que desempeñan, se destacan: la capacidad de acelerar los procesos de descomposición de la materia orgánica, a partir de los hábitos omnívoros y detritívoros de algunas especies (Arias-Pineda y Realpe, 2014; Arroyave-Rincón et al., 2017; Campos, 2014; Yang et al., 2020), su papel como reguladores poblacionales de herbívoros que favorece la producción primaria y secundaria en ambientes marinos y costeros (Giorgini et al., 2019; Gittman y Keller, 2013), a su vez son una fuente de alimento importante para diferentes especies de anfibios, aves, mamíferos y reptiles (Cognetti y Magazzú, 2001; Magalhães, 2001; Rivera-Pérez et al., 2020), son utilizados como bioindicadores de diferentes tipos de contaminación y calidad del agua (Campos y Lasso, 2015; Pinheiro et al., 2021) y también son considerados una base económica importante para las poblaciones humanas costeras (Cardona et al., 2019; Hernández-Maldonado y Campos, 2015). Además, existen especies de interés médico por ser vectores secundarios de tremátodos, como los representantes del género Paragonimus (Phillips et al., 2019) y se destaca la presencia de especies invasoras, que pueden causar importantes desequilibrios en los ecosistemas naturales (Arias-Pineda y Rodríguez, 2012; Camacho-Portocarrero et al., 2021).

Los cangrejos (Meiura) son uno de los grupos más representativos del orden Decapoda, compuesto por los infraórdenes Brachyura y Anomura (Wang et al., 2021; Wolfe et al., 2021). Ambos grupos poseen diez pereópodos (extremidades ambulatorias), de los cua- les los dos primeros están modificados en quelípedos (pinzas). Los Brachyura son conocidos como cangrejos "verdaderos" y se encuentran representantes de las familias Gecarcinidae, Ocypodidae, Portunidae y Pseudothelphusidae (Abele y Kim, 1986; Campos, 2005). Este grupo se caracteriza generalmente por poseer un caparazón oval o subcircular aplanado o ensanchado, un "abdomen" o pleon aplanado que está oculto ventralmente y por presentar la pérdida o reducción significativa de los urópodos (generalmente formando la cola de otros Decapoda). Por otra parte, los Anomura son conocidos como cangrejos "falsos" y están representados por las familias Coenobitidae, Paguridae y Porcellanidae (Martínez-Campos y Campos, 2016). Este segundo infraorden está caracterizado por poseer un caparazón cilíndrico, un abdomen aplanado o poco esclerotizado (en el caso de cangrejos ermitaños), urópodos reducidos y el cuarto par de pereópodos ambulatorios (a veces el tercer) reducido o ausente (García-Raso y Mateo-Ramírez, 2015).

En cuanto a la diversidad, Colombia es el segundo país con mayor diversidad de cangrejos de agua dulce, con cerca de 109 especies (Campos y Campos, 2020; Cumberlidge et al., 2009). La mayoría de las especies presenta distribución reducida, que se restringe a las cadenas montañosas de los Andes, donde encuentran hábitats idóneos (Campos, 2014; Campos y Campos, 2020). En cuanto a los cangrejos semiterrestres y marinos, la información es limitada en muchas áreas del territorio nacional, a pesar de los esfuerzos de instituciones como Invemar, Universidad Nacional, Universidad del Valle, Universidad de Córdoba, Universidad La Salle, Universidad del Atlántico, Universidad de Antioquia y la Universidad del Magdalena, las cuales han elaborado listados de especies, catálogos de distribuciones y claves taxonómicas, con el fin de ampliar el conocimiento en el país (Ardila et al., 2002; Dueñas et al., 2012; GBIF, 2021; 
Martínez-Campos y Campos, 2016; SIB Colombia, 2021).

Los libros rojos de invertebrados terrestres y de cangrejos de agua dulce de Colombia resaltan que estos artrópodos son el tercer grupo más amenazado en el territorio nacional, siendo afectados por la pérdida, fragmentación y degradación del hábitat, como consecuencia de la expansión de las fronteras agropecuarias, los cultivos ilícitos y la sobreexplotación comercial (Amat-García et al., 2007; Campos y Lasso, 2015). Debido a esto y la distribución limitada, la IUCN ha asignado al 42,5\% de las especies en alguna categoría de amenaza, a 28 especies la categoría en Peligro (EN) y 17 en Vulnerable (VU) (Acevedo-Alonso y Cumberlidge, 2021). En cuanto al libro rojo de los invertebrados marinos de Colombia, las especies Mithrax spinosissimus, Carpilius corallinus y Cardisoma guanhumi, distribuidas en el Caribe Colombiano, tienen asignada la categoría de Vulnerable (VU), principalmente por causa de la sobreexplotación pesquera y la perdida de hábitats naturales, llevando una disminución significativa de sus poblaciones naturales (Ardila et al., 2002).

A pesar de la gran diversidad del país, los cangrejos tienen una reducida representatividad en la mayoría de las colecciones de Colombia alcanzando solo el $0,93 \%$ de los registros biológicos del país (SIB Colombia, 2021); esto debido quizás al número limitado de investigadores que trabajan con el grupo y a las pocas faenas de muestreo realizadas. Campos (1985, 2014) afirma que la mayoría de los tipos y el material de referencia de las especies colombianas se encuentran en colecciones del exterior, lo cual dificulta el estudio taxonómico del grupo.

Considerando la importancia ecológica del grupo, los vacíos de información en diferentes áreas del territorio colombiano y sus amenazas, es necesario resaltar la importancia de las colecciones biológicas como patrimonio nacional, ya que constituyen una valiosa fuente de información para potencializar la investigación y conservación de las especies en el país (Fernández et al., 2004; Simmons y Muñoz, 2005). En este sentido, el objetivo principal del presente trabajo es ampliar el conocimiento de los cangrejos (Meiura) mediante la revisión de la Colección Entomológica del Programa de Biología de la Universidad de Caldas (CEBUC).

\section{MATERIALES Y MÉTODOS}

Para el presente estudio se revisaron todos los ejemplares de cangrejos (Meiura) depositados en la Colección Entomológica del Programa de Biología de la Universidad de Caldas (CEBUC). Así mismo, se organizó, sistematizó y determinó todo el material de cangrejos del acervo. El material revisado corresponde a ejemplares obtenidos en el marco de proyectos de investigación, consultorías, donaciones y salidas académicas de la Universidad de Caldas (Manizales). La determinación taxonómica se llevó a cabo con las siguientes claves y descripciones: Abele (1992); Abele y Kim (1986); Campos (2003); Campos (2005); Campos (2014); Coelho y Coelho (1993); De Melo (2003); Dueñas et al. (2012); Karasawa y Schweitzer (2006); Koh y Ng (2008); Masunari y Dubiaski-Silva (1998); Marochi y Masunari (2011); Rodríguez (1982a); Rodríguez (1982b); Sánchez y Campos (1978); Shih et al. (2016); Von Prahl y Manjarrés (1984); así mismo, se consultaron especialistas del grupo a nivel nacional.

Posteriormente, los especímenes fueron fotografiados en vista ventral y dorsal mediante una cámara fotográfica Canon SX530- HS. Se utilizaron los protocolos y metodología para la adecuada preservación de decápodos, según lo sugerido por Rodríguez (1980). La metodología consistió en preservar los especímenes en frascos de vidrio de forma "amanecer", donde los ejemplares con longitud corporal inferior o igual a 2 $\mathrm{cm}$ se fijaron en alcohol al $96 \%$ y los de mayores tamaños se fijaron en una solución de 70/30 de alcohol al $96 \%$ y formol al $4 \%$; igualmente, se realizaron etiquetas con información de recolección y determinación taxonómica de cada espécimen. Adicionalmente, se revisaron las distribuciones registradas en la literatura y en la plataforma GBIF (2021) para las especies encontradas en el trabajo, con el fin de verificar la existencia de nuevos registros. También se compararon los datos de historia natural reportados en la literatura con los datos de recolección (ambiente, hábitat, sustrato, abundancia y estado de desarrollo). Finalmente, por medio del progra- 
ma QGIS Hannover 3.16.3, se produjeron mapas de distribución geográfica de las familias y especies presentes en la colección.

\section{RESULTADOS}

Se encontró un total de 98 individuos de cangrejos (Meiura) representados por 26 hembras (ㅇ), 30 machos $\left(\sigma^{\top}\right)$ y 42 juveniles, pertenecientes a los infraórdenes Brachyura y Anomura, 15 familias, 23 géneros, 22 especies y tres morfoespecies (tabla y figura 1 ). Las familias con un mayor número de individuos registrados en la colección fueron Pseudothelphusidae (exclusiva de agua dulce), seguida por Ocypodidae y Grapsidae (con especies semiterrestres) y Portunidae (familia de especies marinas) (figura 2). Las familias Grapsidae y Pseudothelphusidae presentaron el mayor número de géneros, con tres cada una. Por otra parte, la especie con el mayor número de ejemplares en la colección corresponde a Hypolobocera bouvieri (Rathbun, 1898) de la familia Pseudothelphusidae, con 17 individuos (figura 1A). Cabe mencionar que algunos especímenes no se determinaron a nivel de especie, por contar solamente con hembras o juveniles, lo que imposibilitó la revisión de características diagnósticas del grupo.

\section{Registros}

El $50 \%$ de los ejemplares revisados en la colección CEBUC fueron provenientes del departamento de Caldas y corresponden a la familia Pseudothelphusidae (tabla). Se amplió la distribución de H. bouvieri en el departamento, para las siguientes localidades: La Quinta, municipio de Victoria $\left(5^{\circ} 26^{\prime} 40,0^{\prime \prime} \mathrm{N}\right.$ y $74^{\circ} 54^{\prime} 29,6^{\prime \prime} \mathrm{W} ; 800 \mathrm{~m}$ s. n. m), Rio San Antonio, PNN Selva de Florencia, municipio de Samaná ( $5^{\circ} 30^{\prime} 02,4^{\prime \prime} \mathrm{N}$ y $75^{\circ} 02^{\prime} 56,6^{\prime \prime} \mathrm{W}$; 1020 m s. n. m.) y las quebradas Don Alfonso y Don Fernando, La Flo-
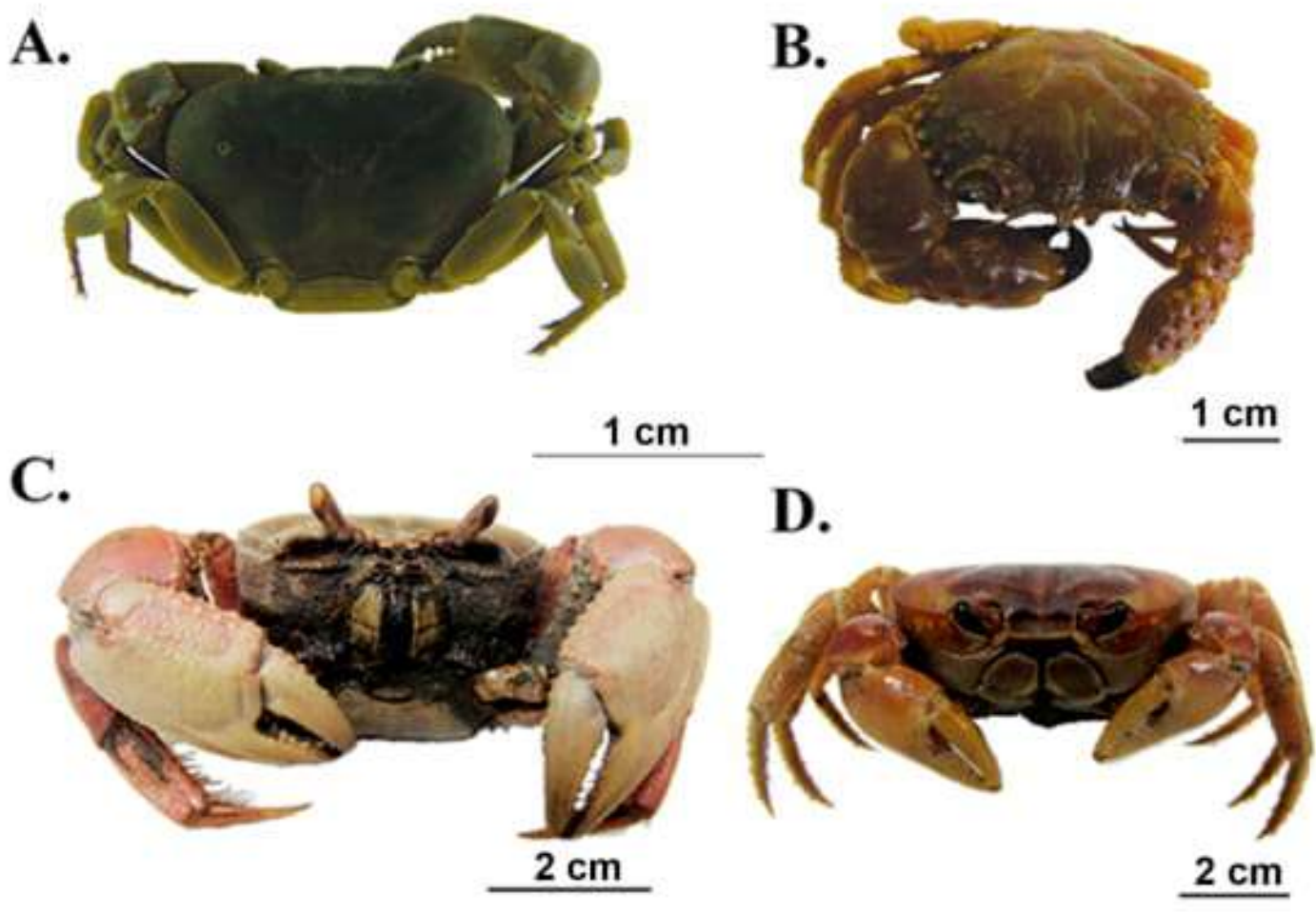

Figura 1. A) Hypolobocera bouvieri (Pseudothelphusidae), B) Eriphia gonagra (Eriphiidae), C) Ucides cordatus (Ocypodidae) y D) Gecarcinus lateralis (Gecarcinidae). 
Tabla. Localidades y tipo de hábitat de los decápodos registrados en la colección CEBUC Universidad de Caldas

\begin{tabular}{|c|c|c|c|c|c|c|c|c|c|}
\hline Orden & Infraorden & Familia & Especies y Morfoespecies & Localidades & Ambiente & Hábitat & Sustrato & Total & $\begin{array}{l}\text { Estado de } \\
\text { desarrollo }\end{array}$ \\
\hline \multirow{25}{*}{ Decapoda } & \multirow{22}{*}{ Brachyura } & \multirow{3}{*}{ Grapsidae } & Geograpsus lividus & Paso Nuevo (Córdoba) & Intermareal & Espolón & Rocoso & 1 & 1 hembra \\
\hline & & & Grapsus grapsus & Paso Nuevo (Córdoba) & Intermareal & Espolón & Rocoso & 3 & $\begin{array}{l}1 \text { hembra, } \\
2 \text { machos }\end{array}$ \\
\hline & & & Goniopsis cruentata & Paso Nuevo (Córdoba) & Estuarino & Manglar & En arboles & 2 & 2 machos \\
\hline & & \multirow{3}{*}{ Ocypodidae } & Minuca rapax & Paso Nuevo (Córdoba) & Terrestre & Manglar & Suelo limoso & 2 & $\begin{array}{l}1 \text { hembra, } \\
1 \text { macho }\end{array}$ \\
\hline & & & Ocypode quadrata & Paso Nuevo (Córdoba) & Terrestre & Playa & $\begin{array}{l}\text { Arena fina y } \\
\text { sedimento }\end{array}$ & 4 & $\begin{array}{l}2 \text { hembras, } \\
2 \text { machos }\end{array}$ \\
\hline & & & Ucides cordatus & Paso Nuevo (Córdoba) & Estuarino & Manglar & Suelo limoso & 1 & 1 hembra \\
\hline & & \multirow[t]{2}{*}{ Gecarcinidae } & Cardisoma guanhumi & Paso Nuevo (Córdoba) & Terrestre & Manglar & Suelo arcilloso & 3 & $\begin{array}{l}2 \text { hembras, } \\
1 \text { macho }\end{array}$ \\
\hline & & & Gecarcinus lateralis & Paso Nuevo (Córdoba) & Terrestre & Espolón & Rocoso & 2 & $\begin{array}{l}1 \text { hembra, } \\
1 \text { macho }\end{array}$ \\
\hline & & \multirow{5}{*}{ Pseudothelphusidae } & Hypolobocera bouvieri & $\begin{array}{l}\text { La Floresta (Caldas), } \\
\text { Selva de Florencia (Caldas), } \\
\text { La Quinta (Caldas) }\end{array}$ & Fluvial & Quebrada & Hojarasca & 17 & $\begin{array}{l}2 \text { machos, } \\
15 \text { juveniles }\end{array}$ \\
\hline & & & Strengeriana florenciae & Selva Florencia (Caldas) & Fluvial & Quebrada & Hojarasca & 10 & $\begin{array}{l}3 \text { machos, } \\
7 \text { juveniles }\end{array}$ \\
\hline & & & Strengeriana maniformis & $\begin{array}{l}\text { Victoria (Caldas), } \\
\text { La Quinta (Caldas) }\end{array}$ & Fluvial & Quebrada & $\begin{array}{l}\text { Vegetación } \\
\text { riparia }\end{array}$ & 15 & $\begin{array}{l}1 \text { macho, } \\
14 \text { juveniles }\end{array}$ \\
\hline & & & Strengeriana fuhrmanni & $\begin{array}{l}\text { Manizales (Caldas), } \\
\text { San José (Caldas) }\end{array}$ & Fluvial & Quebrada & $\begin{array}{l}\text { Vegetación } \\
\text { riparia }\end{array}$ & 5 & $\begin{array}{l}2 \text { machos, } \\
3 \text { juveniles }\end{array}$ \\
\hline & & & Phallangothelphusa dispar & Río Manso (Caldas) & Fluvial & Quebrada & Sedimento fino & 2 & 2 juveniles \\
\hline & & Portunidae & Callinectes ornatus & Paso Nuevo (Córdoba) & Marino & Playa & Fondo arenoso & 7 & $\begin{array}{l}4 \text { hembras, } \\
3 \text { machos }\end{array}$ \\
\hline & & \multirow{2}{*}{ Sesarmidae } & Armases sp. & Paso Nuevo (Córdoba) & Intermareal & Espolón & Rocoso & 1 & 1 juveniles \\
\hline & & & Aratus pisonii & Paso Nuevo (Córdoba) & Intermareal & Espolón & Rocoso & 1 & 1 hembra \\
\hline & & Plagusiidae & Plagusia depressa & Paso Nuevo (Córdoba) & Intermareal & Espolón & Rocoso & 2 & 2 hembras \\
\hline & & Eriphiidae & Eriphia gonagra & Paso Nuevo (Córdoba) & Intermareal & Espolón & Rocoso & 3 & $\begin{array}{l}2 \text { machos, } \\
1 \text { hembra }\end{array}$ \\
\hline & & Trichodactylidae & Sylviocarcinus piriformis & Guayabal (Tolima) & Fluvial & Quebrada & Sedimento fino & 1 & 1 macho \\
\hline & & Calappidae & Calappa cf. sulcata & Paso Nuevo (Córdoba) & Marino & Playa & Fondo arenoso & 1 & 1 macho \\
\hline & & Panopeidae & Eurypanopeus dissimilis & Paso Nuevo (Córdoba) & Intermareal & Espolón & Rocoso & 4 & $\begin{array}{l}2 \text { machos, } \\
2 \text { hembras }\end{array}$ \\
\hline & & Menippidae & Menippe nodifrons & Paso Nuevo (Córdoba) & Intermareal & Playa & Fondo arenoso & 2 & $\begin{array}{l}1 \text { macho, } \\
1 \text { hembra }\end{array}$ \\
\hline & \multirow{3}{*}{ Anomura } & Coenobitidae & Coenobita clypeatus & Paso Nuevo (Córdoba) & Intermareal & Espolón & Rocoso & 3 & $\begin{array}{l}2 \text { machos, } \\
1 \text { hembra }\end{array}$ \\
\hline & & Porcellanidae & Petrolisthes sp. & Paso Nuevo (Córdoba) & Intermareal & Espolón & Rocoso & 4 & $\begin{array}{l}3 \text { hembras, } \\
1 \text { macho }\end{array}$ \\
\hline & & Paguridae & Tomopaguropsis sp. & Paso Nuevo (Córdoba) & Intermareal & Espolón & Rocoso & 2 & 2 hembras \\
\hline Total & 2 & 15 & 25 & & & & & 98 & \\
\hline
\end{tabular}


resta, municipio de Villamaría $\left(5^{\circ} 01^{\prime} 42,1^{\prime \prime} \mathrm{N}\right.$ y $75^{\circ} 31^{\prime} 10,9^{\prime \prime} \mathrm{W} ; 1700 \mathrm{~m}$ s. n. m.), esta última constituye una ampliación de la distribución altitudinal de la especie (figura 3 ).

Adicionalmente, se registra por primera vez para el centro sur del departamento de Caldas, la especie Strengeriana fuhrmanni (Zimmer, 1912) para los municipios de San José $\left(5^{\circ} 05^{\prime} 32,0^{\prime \prime} \mathrm{N}\right.$ y $75^{\circ} 47^{\prime} 46,9^{\prime \prime} \mathrm{W}$; 1650 m s. n. m) y tres localidades de Manizales: Manantial $\left(5^{\circ} 06^{\prime} 12,2^{\prime \prime} \mathrm{N}\right.$ y $75^{\circ} 36^{\prime} 47,6^{\prime \prime} \mathrm{W} ; 1000 \mathrm{~m} \mathrm{s.} \mathrm{n.}$ $\mathrm{m})$, El Águila $\left(5^{\circ} 06^{\prime} 12,2^{\prime \prime} \mathrm{N}\right.$ y $75^{\circ} 30^{\prime} 47,6^{\prime \prime} \mathrm{W} ; 1850$ m s. n. m) y Ecoparque los Yarumos $\left(5^{\circ} 03^{\prime} 40,4^{\prime \prime}\right.$ $\mathrm{N}$ y $75^{\circ} 28^{\prime} 56,1^{\prime \prime} \mathrm{W} ; 2100 \mathrm{~m}$ s. n. m), siendo estas últimas ampliaciones a la distribución altitudinal de la especie (figura 4). Por otro lado, se extendió la distribución en Caldas de la especie $S$. florenciae Campos, 1995 (localidad: Rio San Antonio, PNN Selva de Florencia, Samaná $-5^{\circ} 30^{\prime} 02,4^{\prime \prime}$ N y $75^{\circ} 02^{\prime} 56,6^{\prime \prime} \mathrm{W}$; 1020 m. s. n. m.) y S. maniformis Campos \& Rodríguez, 1993 (dos localidades de Victoria $-5^{\circ} 26^{\prime} 40,0^{\prime \prime}$ N y $74^{\circ} 54^{\prime} 29,6^{\prime \prime} \mathrm{W} ; 800$ m. s. n. m y $5^{\circ} 19^{\prime} 43,9^{\prime \prime}$ $\mathrm{N}$ y $74^{\circ} 55^{\prime} 10,3^{\prime \prime} \mathrm{W} ; 1000$ m. s. n. m) (figura 4). Por último, también se amplió el rango de distribución en
Caldas de la especie Phallangothelphusa dispar (Zimmer, 1912), en la localidad de Reserva Rio Manso, Norcasia $\left(5^{\circ} 39^{\prime} 59,0^{\prime \prime} \mathrm{N}\right.$ y $74^{\circ} 46^{\prime} 51,9^{\prime \prime} \mathrm{W} ; 228 \mathrm{~m}$ s. n. m) (figura 5).

El departamento de Córdoba fue el segundo departamento con más registros en la colección CEBUC, en donde todos los especímenes capturados correspondieron a la localidad de Paso Nuevo, municipio de San Bernardo del viento $\left(9^{\circ} 19^{\prime} 51,6^{\prime \prime} \mathrm{N}\right.$ y $76^{\circ} 05^{\prime} 07,0^{\prime \prime} \mathrm{W} ; 0 \mathrm{~m}$ s. n. m.). En esta localidad se registró también la mayor riqueza de cangrejos (19 especies), siendo estos representantes marinos y semiterrestres en diferentes microhábitats (tabla), pertenecientes a las familias: Gecarcinidae, Grapsidae, Ocypodidae, Plagusiidae y Sesarmidae (figura 6); Portunidae, Eriphiidae, Calappidae, Panopeidae y Menippidae pertenecientes al infraorden Brachyura (figura 7); y Coenobitidae, Porcellanidae, Paguridae del infraorden Anomura (figura 8). Finalmente, se amplió la distribución de la especie Sylviocarcinus piriformis (Pretzmann, 1968) de la familia Trichodactylidae, en el departamento del Tolima, para el municipio de Armero Guayabal $\left(5^{\circ} 00^{\prime} 29,4^{\prime \prime} \mathrm{N}\right.$ y

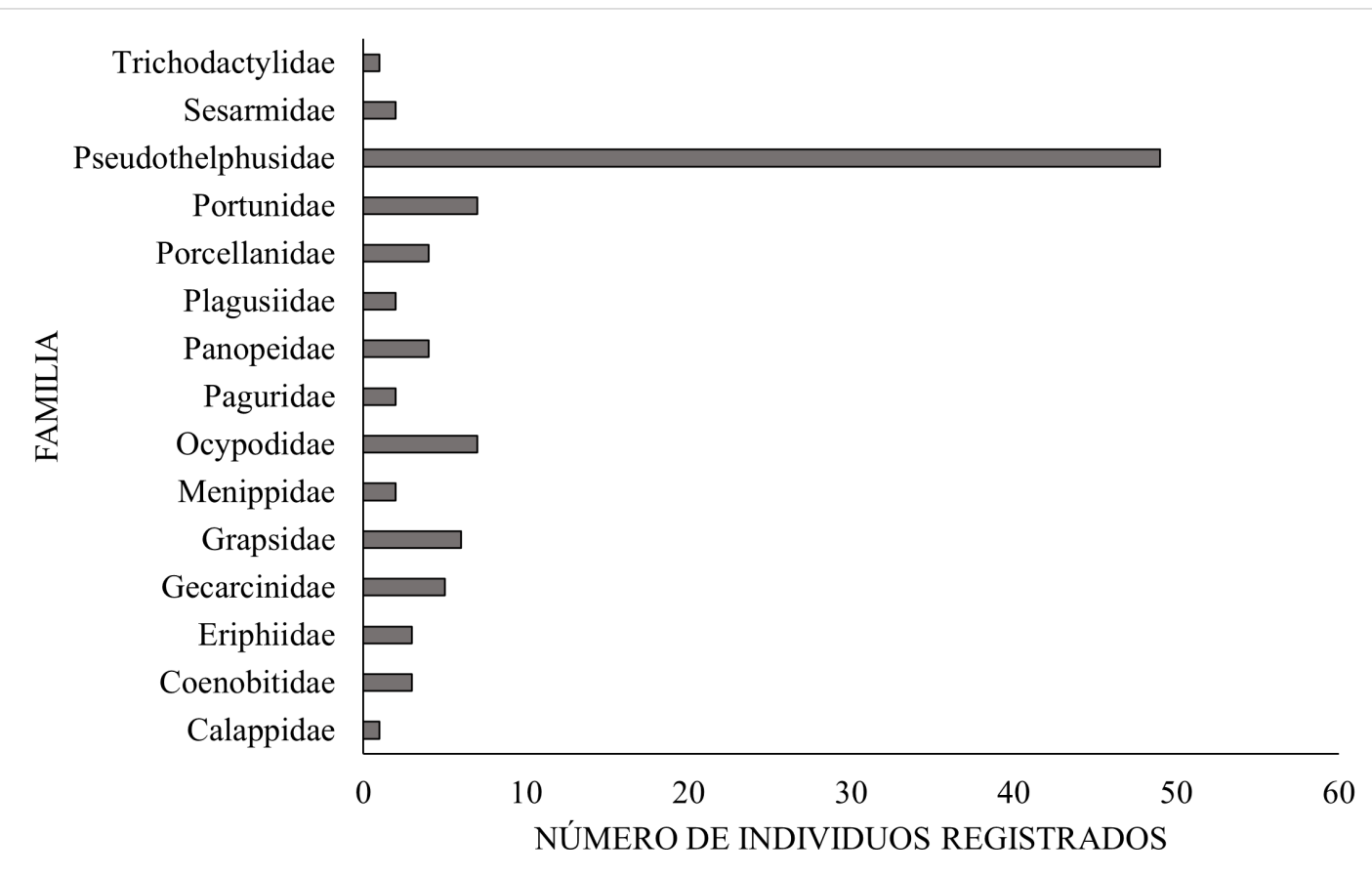

Figura 2. Número de individuos registrados por familia de Decapoda en la Colección Entomológica del Programa de Biología de la Universidad de Caldas (CEBUC). 
$74^{\circ} 53^{\prime} 48,3^{\prime \prime} \mathrm{W} ; 280$ m s. n. m) (figura 5).

\section{DISCUSIÓN}

En este trabajo se registró a $S$. fuhrmanni por primera vez en el centro sur de Caldas y se ampliaron los rangos de distribución para otras cuatro especies de cangrejos de agua dulce de la familia Pseudothelphusidae en este departamento; además, se documentaron 19 especies de cangrejos marinos y semiterrestres para el municipio de San Bernardo del viento en Córdoba y una especie de la familia Trichodactylidae para el departamento del Tolima. A pesar de que las especies de CEBUC representan solo el 2,17\% de los decápodos de Colombia (SIB Colombia, 2021), los nuevos registros documentados proporcionan información importante para futuros estudios en taxonomía, ecología, diversidad y con- servación del grupo en la región central y el caribe colombiano (Simmons y Muñoz, 2005).

El mayor número de taxones encontrados corresponde a la familia Pseudothelphusidae (figura 2), la cual es una familia de amplia distribución en el neotrópico, con un extenso rango altitudinal desde los cero hasta los 3000 m s. n. m. (Campos, 2005; Campos y Pedraza, 2007; Rodríguez, 1981, 1982b). La dominancia de pseudotelfúsidos en la colección se puede explicar por hábitos ecológicos y por el enfoque de investigación de los profesionales relacionados con la colección CEBUC, es decir, los ejemplares fueron recolectados en proyectos limnológicos o en salidas académicas de campo, donde se recolectaron macroinvertebrados acuáticos en biotopos típicos de estos cangrejos, como son la vegetación ribereña y el sustrato rocoso (Campos y Rodríguez, 1985; Campos, 2000; Campos y Campos, 2020).
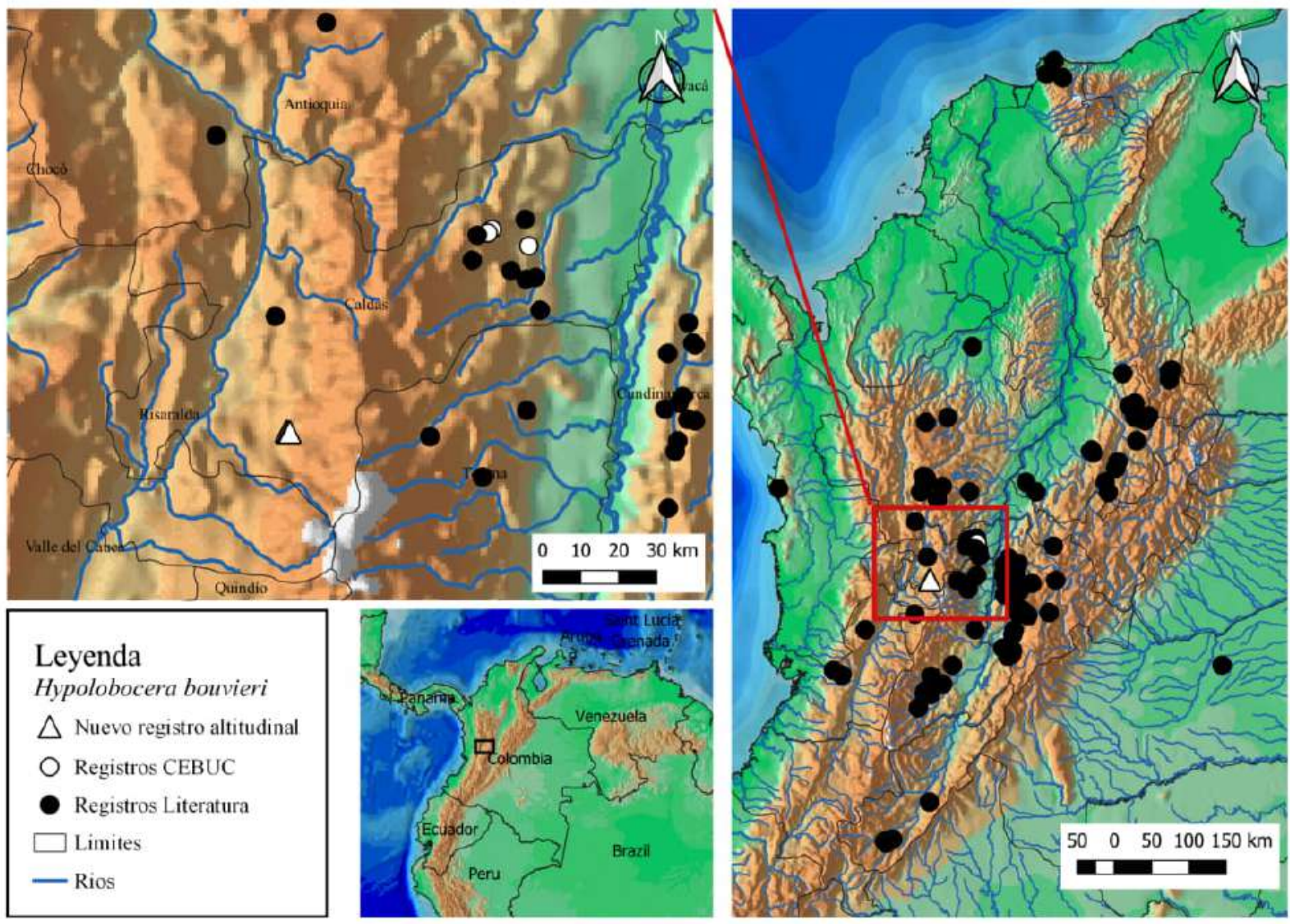

Figura 3. Mapa de la distribución geográfica de Hypolobocera bouvieri (Pseudothelphusidae), con ampliación en el rango de distribución en Caldas. 


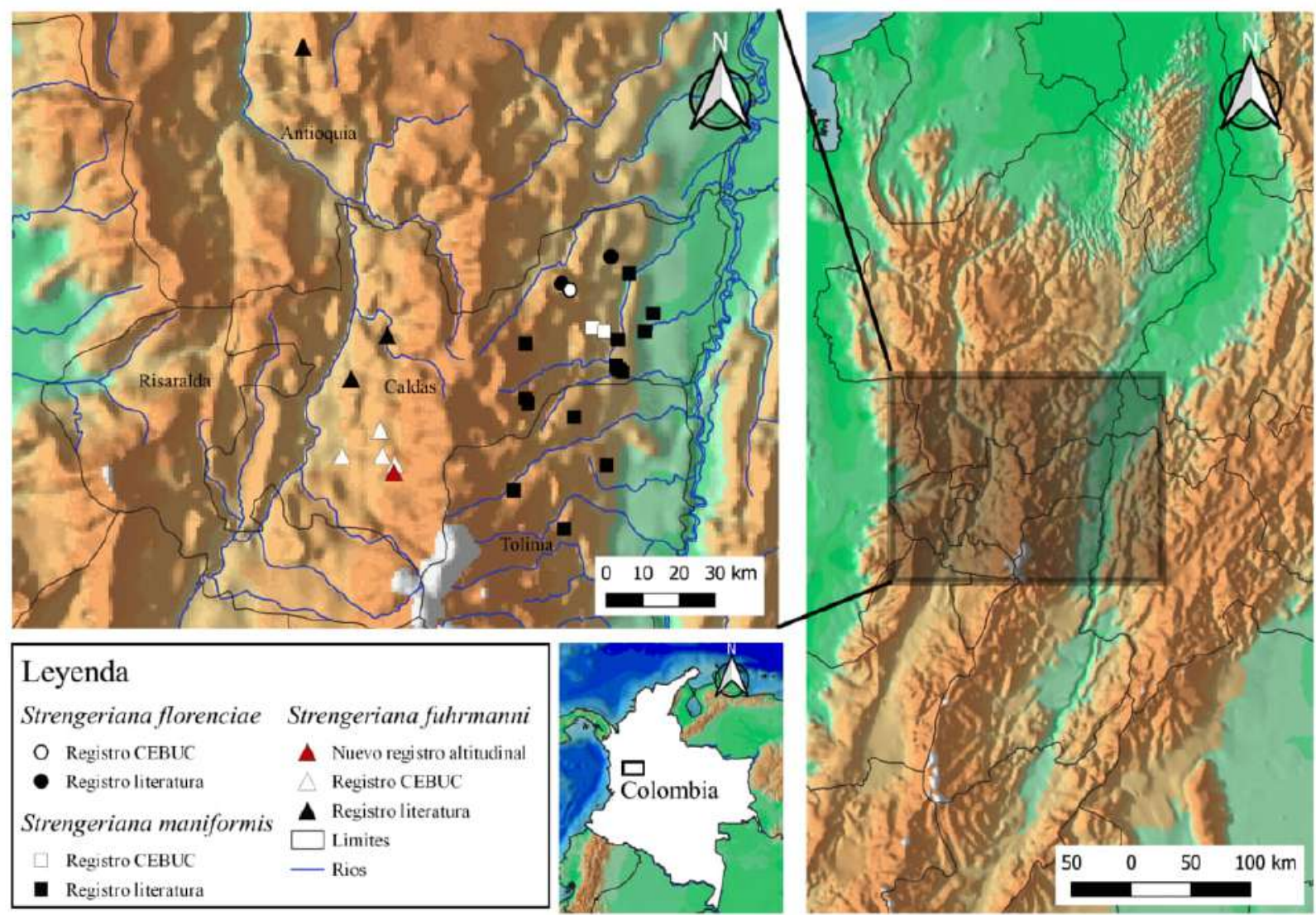

Figura 4. Mapa de la distribución geográfica de Strengeriana florenciae, Strengeriana maniformis y Strengeriana fuhrmanni (Pseudothelphusidae), con ampliación en el rango de distribución en Caldas.

Los registros de $H$. bouvieri en la región montañosa de Caldas representan una ampliación de la distribución altitudinal de esta especie de los $1100 \mathrm{~m}$ s. n. m. hasta los 1700 m s. n. m. Esta especie posee una extensa distribución en Colombia, desde la Sierra Nevada de Santa Marta hasta los valles de las Cuencas de los ríos Cauca y Magdalena (Campos, 2005, 2014). Igualmente, se amplió la distribución altitudinal de S. fuhrmanni (Zimmer, 1912), distribuida anteriormente en la Cuenca medio del río Cauca desde el sur Antioquia y norte de Caldas con altitudes máximas de 1790 m s. n. m. (Campos, 2005, 2014) y ahora hasta el sur occidente del departamento de Caldas hasta los $2100 \mathrm{~m} \mathrm{s.} \mathrm{n.} \mathrm{m.}$

Los registros aquí documentados para $H$. bouvieri y S. fuhrmanni en el departamento de Caldas constituyen una base importante en el conocimiento de su distribución en la ecorregión de la cordillera Central,
Río Cauca (Campos y Campos, 2020). Muchas de las nuevas localidades reportadas en este trabajo fueron en quebradas con una importante presión antrópica, como es el caso de los registros de $H$. bouvieri que fueron capturados en el municipio de Villamaría-Caldas (vereda La Floresta), en una quebrada ubicada en una matriz agrícola con horticultura de montaña. El manejo de estos cultivos es convencional con uso de pesticidas y fertilizantes (Nicholls, 2008). Por otro lado, S. fuhrmanni fue recolectado en el perímetro urbano Manizales-Caldas, donde se presentan matrices urbanizadas con vegetación riparia (Tobasura, 2008). Estas afectaciones en la integridad de las quebradas representan una amenaza para las poblaciones de cangrejos que habitan estos cuerpos de agua, debido al rápido crecimiento poblacional y desarrollo agropecuario en la región Andina (Acevedo-Alonso y Cumberlidge, 2021; Campos y Lasso, 2015). A mediano y largo plazo, estas amenazas junto con el 


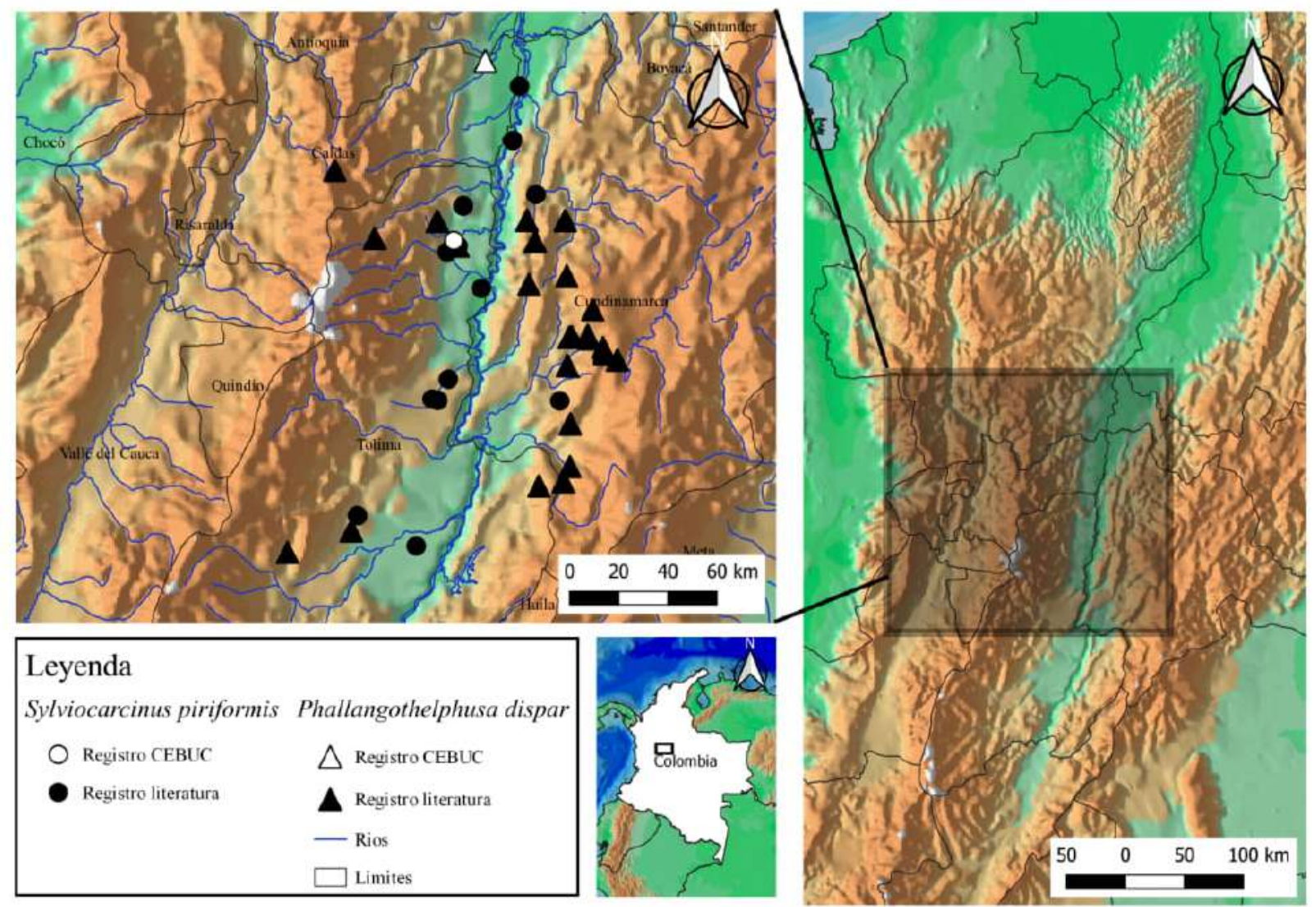

Figura 5. Mapa de la distribución geográfica de Sylviocarcinus piriformis (Trichodactylidae) y Phallangothelphusa dispar (Pseudothelphusidae), con ampliación de la distribución en los departamentos de Caldas y Tolima.

aumento de la temperatura a causa del calentamiento global generan nuevos desafíos y situaciones críticas para muchas de las especies endémicas de esta región (Hudson et al., 2021).

Los especímenes encontrados en el departamento de Córdoba estuvieron asociados con hábitats altamente transformados, donde se encontraron fragmentos de bosque de manglar con las especies Rhizophora mangle, Avicennia germinans y Laguncularia racemosa C.F. La presencia de dichas especies vegetales, en la zona estudiada, son factores fundamentales para la persistencia de las especies de cangrejo Goniopsis cruentata Latreille, 1803, Minuca rapax (Smith, 1870), Ucides cordatus (Linnaeus, 1763) y Cardisoma guanhumi Latreille, 1825 (Quirós y Campos, 2010). Al igual en playas con una plataforma continental con abundantes formaciones rocosas, en donde se registraron Ocypode quadrata Fabricius, 1787, Callinectes ornatus (Ordway, 1863), Calappa cf. sulcata Rathbun, 1898 y Menippe nodifrons Stimpson, 1859. Estos hábitats, acompañados de orillas de ríos con abundante hojarasca, sustratos arenosos y vegetación nativa, proporcionan a las especies multitud de hábitats idóneos para su desarrollo (RangelBuitrago y Posada-Posada, 2005). Cabe destacar que las especies encontradas en esta zona son claves en la alimentación de comunidades humanas costeras, como es el caso de los géneros Cardisoma, Callinectes, Calappa y Ucides (Cardona et al., 2019; Fischer et al., 1995); además, son fundamentales en las redes tróficas (Pinheiro et al., 2021) e importantes para evaluar la perturbación humana en ecosistemas costeros (Novais et al., 2021). 

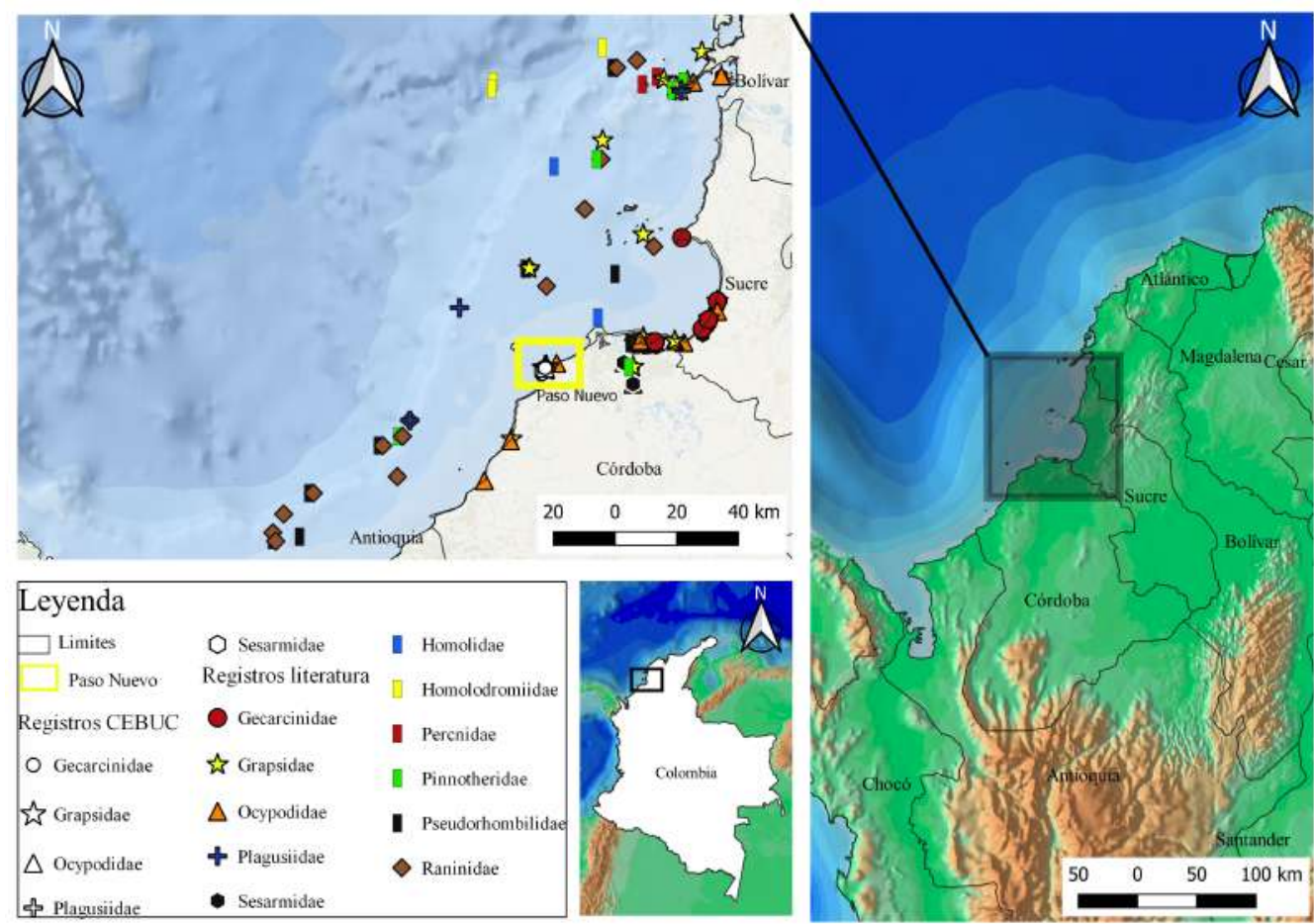

Figura 6. Mapa de la distribución geográfica de familias de Brachyura no Heterotremata (Decapoda: Meiura) marinos y semiterrestres asociados a la ecorregión del Golfo de Morrosquillo, con nuevos registros para el sector Paso Nuevo (San Bernardo del Viento).

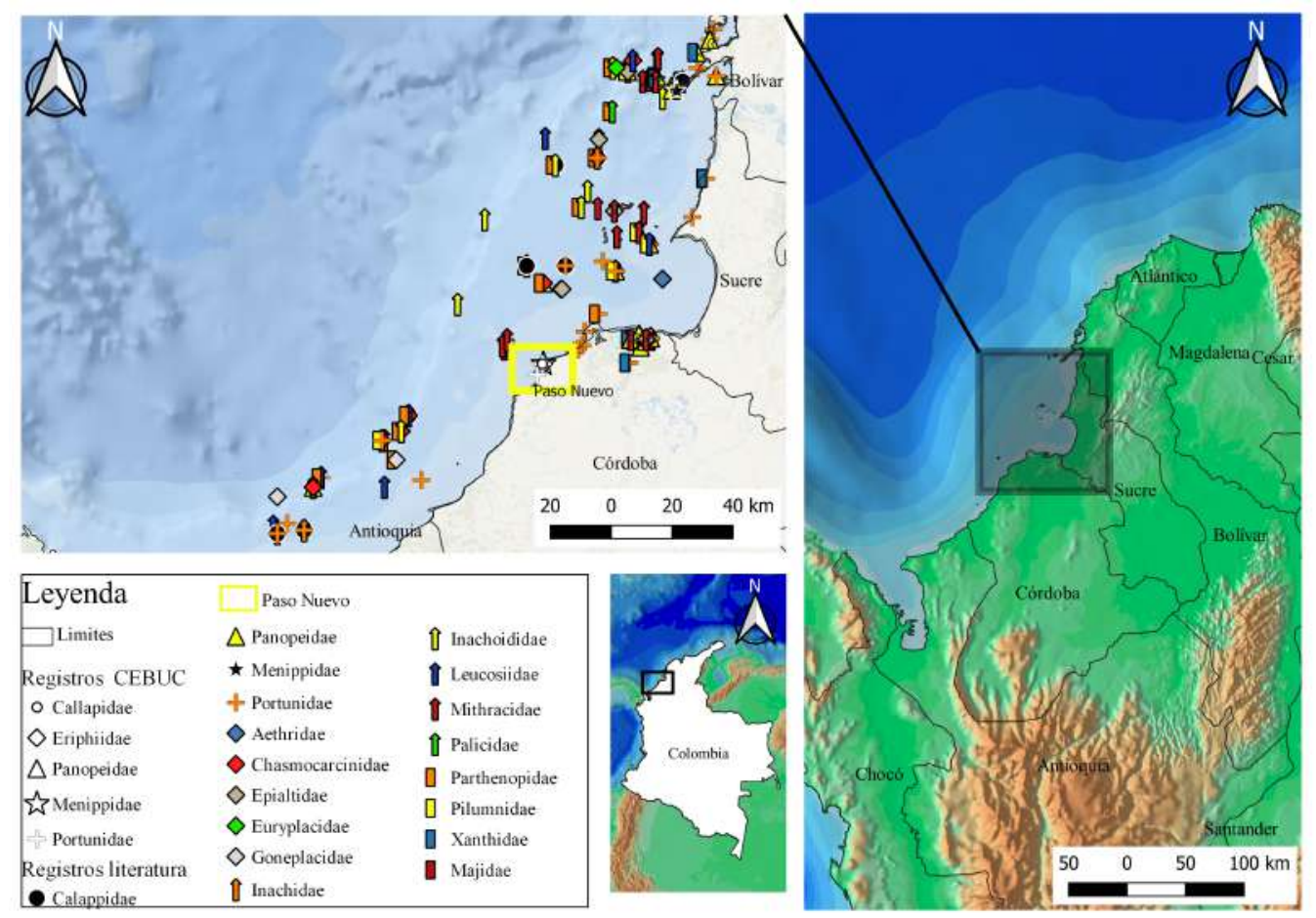

Figura 7. Mapa de la distribución geográfica de familias de Brachyura Heterotremata (Decapoda: Meiura) marinos y semiterrestres asociados a la ecorregión del Golfo de Morrosquillo, con nuevos registros para el sector Paso Nuevo (San Bernardo del Viento). 
El $60 \%$ de los individuos revisados de la localidad Paso Nuevo (Córdoba) fueron recolectados en "espolones", que son muros rocosos usados para aminorar el impacto de las olas en la playa (Rangel-Buitrago y Posada-Posada, 2005) y que generan hábitats para algas, moluscos, crustáceos y que son ricos en alimento y zonas de refugio (Díaz-Pulido, 2001). En los espolones se recolectaron las especies Geograpsus lividus (H. Milne Edwards, 1837), Grapsus grapsus Linnaeus, 1758, Armases sp., Aratus pisonii (H. Milne Edwards, 1837), Plagusia depressa (Fabricius, 1775), Eriphia gonagra (Fabricius, 1781), Eurypanopeus dissimilis (Benedict \& Rathbun, 1891), Gecarcinus lateralis (Freminville, 1835), Coenobita clypeatus (Fabricius, 1787), Petrolisthes sp. y Tomopaguropsis sp. Este último hábitat, a pesar de tener muchas veces un origen antrópico, presenta una gran diversidad de cangrejos, ya que posee condiciones adecuadas para algunas especies (Quirós et al., 2012). Para el caso de cangrejos ermitaños (Coenobitidae y Paguridae), los espolones permiten la acumulación de materia, como conchas de gasterópodos, fundamentales como refugios móviles de estas especies, donde el aumento de este material puede incrementar la abundancia de estos organismos (Hahn, 1998).

Finalmente, en este trabajo el departamento del Tolima está solamente representado en la colección por la localidad de Armero-Guayabal, donde se registró S. piriformis (Pretzmann, 1968). Esta localidad se encuentra ubicada dentro de la granja experimental de la Universidad del Tolima, constituida por una matriz mixta de cultivos rodeada por cuerpos de agua, dando como resultado microhábitats donde se pueden establecer estos organismos (Campos y Lasso, 2015). Se destaca que este punto corresponde a una nueva localidad para la especie en el departamento, antes reportada en Coello y Mariquita (Campos, 2005). El estado de vulnerabilidad de "grado de menor preocupación" (LC) de esta especie en la lista roja de la IUCN, quizás sea debido a su amplia distribución y su aparente tolerancia a la

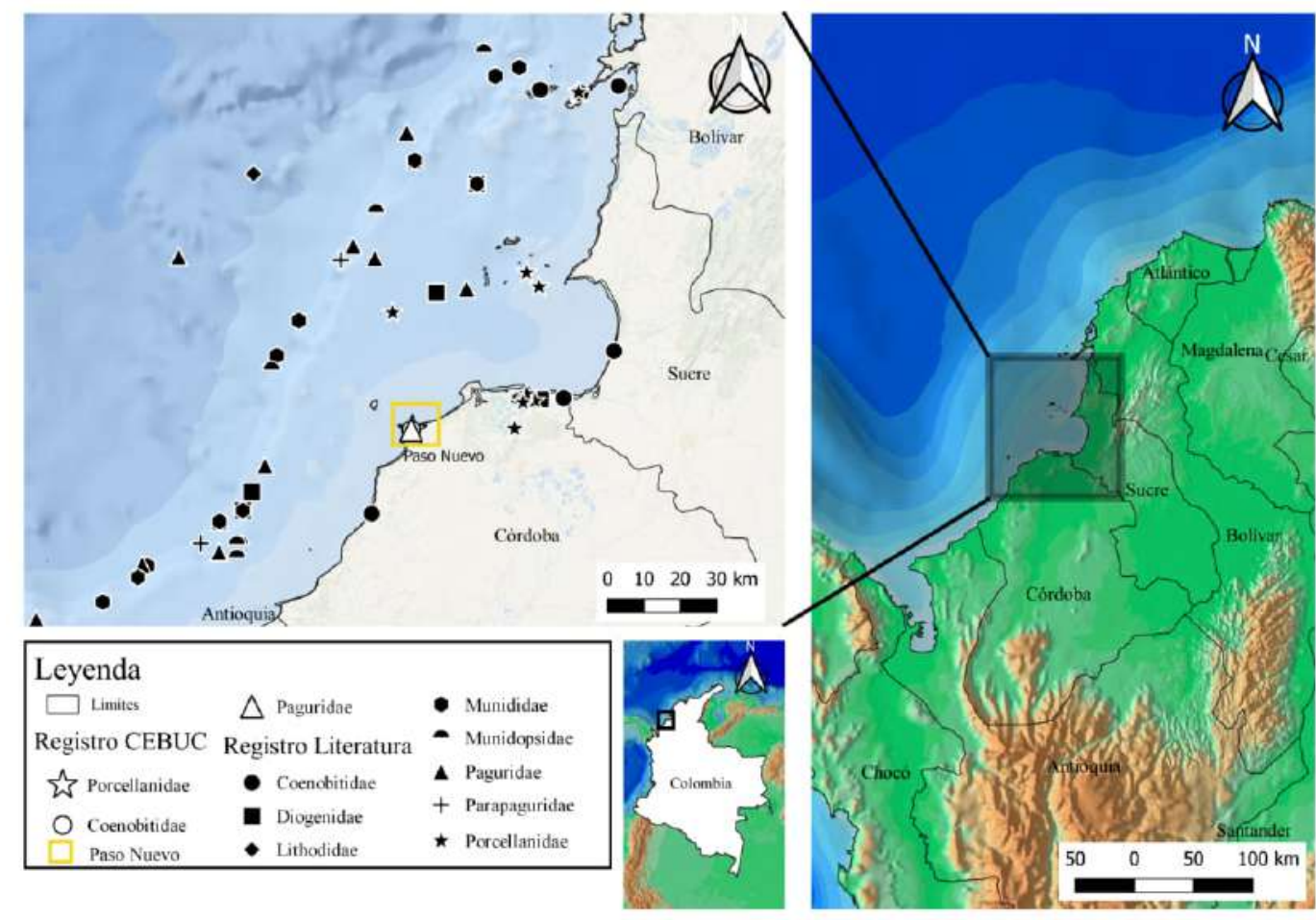

Figura 8. Mapa de la distribución geográfica de familias de Anomura (Decapoda: Meiura) marinos y semiterrestres asociados a la ecorregión del Golfo de Morrosquillo, con nuevos registros para el sector Paso Nuevo (San Bernardo del Viento). 
modificación del hábitat. Sin embargo, el incremento de la contaminación en los cuerpos de agua puede ser una amenaza a sus poblaciones (Cumberlidge, 2008).

También queremos resaltar el valor de las colecciones biológicas como fuente primaria de información para estimar la diversidad, distribución de este grupo y, para futuros estudios en biología molecular, biogeografía, conservación, taxonomía y modelaje (Segadilha y Rodrigues, 2020). Se resalta la necesidad de revisar las colecciones regionales y aumentar los esfuerzos de muestreo en el país, para con ello generar listados taxonómicos, guías de identificación accesibles y claves taxonómicas especializadas que permitan ampliar, identificar, determinar y evaluar la diversidad de cangrejos en el país en escenarios futuros de perdida de la biodiversidad.

Finalmente, la información generada a partir de la revisión del material biológico de la colección CEBUC amplía la distribución y aporta nuevas localidades de diferentes especies de cangrejos (Meiura) en Colombia, mejorando los datos existentes de historia natural, biología y distribución para futuras investigaciones, con ello mostrando el potencial de generar nuevo conocimiento con base en el material disponible en las colecciones. Así mismo, esta información es importante como insumo para la toma de decisiones relacionado con el desarrollo de áreas de importancia biológica para la conservación de cangrejos (Meiura) en el territorio nacional.

\section{AGRADECIMIENTOS}

Los autores agradecemos a la Colección Entomológica del Programa de Biología de la Universidad de Caldas (CEBUC) y al Grupo Biodiversidad y Recursos Naturales (Bionat) por disponer equipos e infraestructura para la identificación del material y el análisis de los datos. A la comunidad de Paso Nuevo, San Bernardo del Viento (Córdoba), y a Martha Campos por la confirmación de los especímenes de la familia Pseudothelphusidae. A los revisores anónimos por su acertada contribución al manuscrito.

\section{CONFLICTO DE INTERESES}

Los autores declaran no tener conflictos de intereses en el presente trabajo.

\section{REFERENCIAS}

Abele, L. G. (1992). A review of the grapsid crab genus Sesarm (Crustacea: Decapoda: Grapsidae) in America, with the description of a new genus. Smithsonian Contributions to Zoology, 1(1), 1-57. DOI:10.5479/si.00810282.527

Abele, L. G., \& Kim, W. (1986). An illustrated guide to the marine decapod crustaceans of Florida (Technical series $8(1)$, part 2). State of Florida, Department of Environmental Regulation. https://decapoda.nhm.org/pdfs/ 12365/12365-001.pdf

Acevedo-Alonso, A., \& Cumberlidge, N. (2021). Updated extinction risk assessment of the Colombian freshwater crabs (Brachyura: Pseudothelphusidae, Trichodactylidae) reveals an increased number of threatened species. In $\mathrm{T}$. Kawai., \& D. C. Rogers, Recent Advances in Freshwater Crustacean Biodiversity and Conservation (pp. 405-424). CRC Press. DOI:10.1201/9781003139560

Amat-García, G., Amat-García, E., Andrade-C, M. G., \& Rodríguez-Mahecha, J. V. (Eds.). (2007). Libro rojo de los invertebrados terrestres de Colombia. Conservación Internacional Colombia, Instituto Alexander von Humboldt, Ministerio de Ambiente, Vivienda y Crédito Territorial.

Ardila, N., Navas, G. R., \& J. Reyes, J. (Eds.). (2002). Libro rojo de invertebrados marinos de Colombia. INVEMAR. Ministerio de Medio Ambiente.

Arias-Pineda, J. Y., \& Rodríguez, W. D. (2012). New record of the invasive species Procambarus (Scapulicambarus) clarkia (Girard 1852) (Crustacea, Decapoda, Cambaridae) from the Colombian Eastern Cordillera. Boletín de la Sociedad Entomológica Aragonesa (S.E.A), 51(1), 313- 315. http://seaentomologia.org/PDF/Boletin51/313315BSEA51NBPho ronProcambarusCordorientalColombiacolor .pdf

Arias-Pineda, J. Y., \& Realpe, E. (2014). Ampliación de la distribución conocida del cangrejo sabanero, Neostrengeria macropa (Milne- Edwards, 1853) (Decapoda: Pseudothelphusidae), en la sabana de Bogotá (Colombia). Boletín de la Sociedad Entomológica Aragonesa (S.E.A), 55, 141-146. http://sea-entomologia. org/Publicaciones/Boletines/Boletin55/Boletin55/ 141146BSEA55Cangrejosabanero.pdf

Arroyave-Rincón, A., Amórtegui-Torres, V., Blanco-Libreros, J. F., \& Taborda-Marín, A. (2017). Efecto de borde sobre la población del cangrejo azul Cardisoma guanhumi; (Decapoda: Gecarcinidae) en el manglar de la bahía El Uno, golfo de Urabá (Colombia): una aproximación a su captura artesanal. Actualidades Biológicas, 36(100), 47-57. https://revistas.udea.edu.co/index.php/ actbio/article/view/331377

Camacho-Portocarrero, R. F., Duarte-Gándica, I., \& Altamiranda-Saavedra, M. (2021). Áreas en riesgo 
de invasión por Procambarus clarkii (Decapoda: Cambaridae), un cangrejo de río introducido en Colombia. Revista de Biología Tropical, 69(1), DOI:10.15517/RBT.V69I1.41493

Campos, M. R. (1985). Decápodos de agua dulce del Suborden Brachyura reportados para Colombia. Caldasia, 14(67), 265-284. https://www.jstor.org/stable/43405956

Campos, M. R. (2000). Neostrengeria binderi, a new species of pseudothelphusid crab from the eastern Andes of Colombia (Crustacea: Decapoda: Brachyura). Proceedings of the Biological Society of Washington, 105(3), 540-554. https://decapoda.nhm.org/pdfs/31177/31177.pdf

Campos, M. R. (2003). A review of the freshwater crabs of the genus Hypolobocera Ortmann, 1897 (Crustacea: Decapoda: Brachyura: Pseudothelphusidae), from Colombia. Proceedings Biological Society of Washington, 116(3), 754-802. https://decapoda.nhm.org/pdfs/ 31180/31180.pdf

Campos, M. R. (2005). Freshwater crabs from Colombia. A taxonomic and distributional study. Academia Colombiana de Ciencias Exactas, Físicas y Naturales. http: //repositorio.accefyn.org.co//handle/001/108

Campos, M. R. (2014). Crustáceos decápodos de agua dulce de Colombia. Universidad Nacional de Colombia.

Campos, M. R. \& Campos, D. (2020). Distribution and species diversity of freshwater crabs of the family Pseudothelphusidae in Colombia (Crustacea: Decapoda: Brachyura). Nauplius, 28(1), e2020036. DOI:10.1590/2358$2936 \mathrm{e} 2020036$.

Campos, M. R. \& Lasso C. A. (2015). Libro rojo de los cangrejos dulceacuícolas de Colombia. Instituto de Investigación de Recursos Biológicos Alexander von Humboldt (IAvH), Universidad Nacional de Colombia. http://www. humboldt.org.co/es/estado-de-los-recursos-naturales/ item/833-libro-rojo-cangrejos

Campos, M. R. \& Pedraza., M. (2007). Estudio de la variación morfológica del gonópodo de Strengeriana maniformis (Brachyura: Pseudothelphusidae) mediante aplicación de morfometría geométrica. Caldasia, 29(1), 143-152. https: //repositorio.unal.edu.co/handle/unal/72941

Campos, M. R. \& Rodríguez, G. (1985). A new species of Neostrengeria (Crustacea, Decapoda, Pseudothelphusidae) with notes on the geographical distribution of the genus. Proceedings of the Biological Society of Washington, 98(1), 718-727. https://decapoda.nhm.org/pdfs/27412/ 27412.pdf

Cardona, L. F., Campos, N. H., \& Rolong, E. H. (2019). Parámetros de crecimiento de Cardisoma guanhumi en el departamento del Magdalena, Colombia. Boletín de Investigaciones Marinas y Costeras, 48(1), 27-41. DOI:10.25268/bimc.invemar.2019.48.1.755

Coelho, P. A. \& Coelho Filho, P. A. (1993). Proposta de classificação da família Xanthidae (Crustacea, Decapoda, Brachyura) através da taxonomia numérica. Revista Brasiliera de Zoologia, 10(4), 559-580. DOI:10.1590/S010181751993000400001.

Cognetti, G., M. Sara \& Magazzú, G. (2001). Biología marina. Editorial Ariel.

Cumberlidge, N. (2008). Sylviocarcinus piriformis. The IUCN Red List of Threa- tened Species 2008:e.T134675A3996516. DOI:10.2305/IUCN.UK.2008.RLTS

Cumberlidge, N., Ng, P. K., Yeo, D. C., Magalhães, C., Campos, M. R., Alvarez, F., Naruse, T., Daniels, S. R., Esser, L., Attipoe, F., Cotilde-Ba, F., Darwall, W., Mclvor, A., Baillie, J., Ram, M., \& Ram, M. (2009). Freshwater crabs and the biodiversity crisis: importance, threats, status, and conservation challenges. Biological Conservation, 142(8), 1665-1673. DOI:10.1016/j.biocon.2009.02.038.

De Melo, G. A. S. (2003). Manual de identificação dos Crustacea Decapoda de água doce do Brasil. Edições Loyola.

Díaz-Pulido, G. (2001). Litorales marinos y costeros Programa de Biodiversidad y Ecosistemas Marinos. Informe Nacional sobre el estado de la biodiversidad en Colombia (pp. 115-128). INVEMAR.

Dueñas, P. R., Campos, N. H., \& Quirós, J. A. (2012). Los crustáceos decápodos del departamento de Córdoba, Colombia: Biodiversidad de la fauna de crustáceos decápodos en el Caribe colombiano. Editorial Académica Española.

Fernández, G., Sosa, V., León, J., \& León C. (2004). Colecciones biológicas. Centros de Investigación. CONACYT. ECOSUR.

Fischer, W., Krupp, F., Schneider, W., Sommer, C., \& Carpenter, K. E. (1995). Guía FAO para la identificación de especies para los fines de la pesca: Pacífico centrooriental. (Vol. I Plantas e Invertebrados). FAO. http: //www.fao.org/3/t0851s/t0851s00.htm

García-Raso, J. E., \& Ramírez, A. M. (2015) Orden Decapoda. Manual. Boletín de la Sociedad Entomológica Aragonesa (S.E.A) 80(1), 117. http://sea-entomologia.org/IDE@ /revista_80.pdf

GBIF (2021, abril 11). Global Biodiversity Information Facility. Occurrence Download. DOI:10.15468/dl.cgrh48

Giorgini, M., Miguez, A., Esquius, K. S., de Astarloa, C. D., Iribarne, O., Fanjul, E., \& Escapa, M. (2019). Regenerative bioturbation by intertidal burrowing crabs modifies microphytobenthic composition and enhances primary production in SW Atlantic mudflats. Marine Ecology Progress Series, 632, 43-57. DOI:10.3354/meps13134

Gittman, R. K., \& Keller, D. A. (2013). Fiddler crabs facilitate Spartina alterniflora growth, mitigating periwinkle overgrazing of marsh habitat. Ecology, 94(12), 2709-2718. DOI:10.1890/13-0152.1

Hahn, D. R. (1998). Hermit crab shell use patterns: response to previous shell experience and to water flow. Journal of Experimental Marine Biology and Ecology, 228(1), 35-51. DOI:10.1016/S0022-0981(98)00002-1

Hernández-Maldonado, A., \& Campos, N. H. (2015). Estado actual de la población adulta del cangrejo semiterrestre Cardisoma guanhumi (Latreille) en la isla de San Andrés, Caribe Colombiano. Boletín de Investigaciones Marinas y Costeras, 44(1), 185-198. https://aquadocs.org/handle/ $1834 / 8105$

Hudson, D. M., Phillips, G., Lasso, C. A., \& Campos, M. R. (2021). Threats to Endemic Colombian Freshwater Crabs (Decapoda: Pseudothelphusidae, Trichodactylidae) Associated with Climate Change and Human-Mediated Activities. In T. Kawai, \& D. Christopher Rogers, Recent Advances in Freshwater Crustacean Biodiversity and Conservation. CRC Press. 
Karasawa, H., \& Schweitzer, C. E. (2006). A new classification of the Xanthoidea sensu lato (Crustacea Decapoda: Brachyura) based on phylogenetic analysis and traditional systematics and evaluation of all fossil Xanthoidea sensu lato. Contributions to Zoology, 75(1/2). DOI:10.1163/18759866-0750102002

Koh, S. K., \& Ng, P. K. (2008). A revision of the shore crabs of the genus Eriphia (Crustacea: Brachyura: Eriphiidae). Raffles Bulletin of Zoology, 56(2), 327-355. http://scholarbank.nus.edu.sg/handle/10635/99954

Magalhães, C. (2001). Diversity, distribution, and habitats of the macro-invertebrate fauna of the Rio Paraguay and Rio Apa, Paraguay, with emphasis on Decapod Crustaceans. In C. Barry, P.W. Willink, \& J.R. Montambault, A Biological Assessment of the Aquatic Ecosystems of the Rio Paraguay Basin, Alto Paraguay, Paraguay. Conservation International. RAP Bulletin of Biological Assessment, 19(1), 68-72.

Marochi, M. Z., \& Masunari, S. (2011). Os caranguejos Eriphiidae, Menippidae, Panopeidae e Pilumnidae (Crustacea Brachyura) de águas rasas do litoral do Paraná, com chave pictórica de identificação para as espécies. Biota Neotropica, 11 (3), 21-33. DOI:10.1590/S167606032011000300001.

Martínez-Campos, B., \& Campos, N. H. (2016). Registros preliminares del "Catálogo de cangrejos ermitaños del mar Caribe colombiano". v1.0. Instituto de Investigaciones Marinas y Costeras INVEMAR. DOI:10.15472/pya8vr

Masunari, S., \& Dubiaski-Silva, J. (1998). Crustacea decapoda da praia rochosa da Ilha do Farol, Matinhos, Paraná: II. Distribuição espacial de densidade das populações. Revista Brasileira de Zoologia, 15(3), 643-664. DOI:10.1590/S0101-8175199800030001.

Nicholls, C. 1. (2008). Control biológico de insectos: un enfoque agroecológico. Universidad de Antioquia.

Novais, W., Carvalho, F., \& Couto, E. (2021). Conservation of the endangered blue land crab Cardisoma guanhumi Latreille in Latreille, Le Peletier, Serville \& Guérin, 1828 (Decapoda: Brachyura: Gecarcinidae) in Brazil: optimal habitats and environmental factors. Journal of Crustacean Biology, 41(2), ruab011. DOI:10.1093/jcbiol/ruab011

Phillips, G., Hudson, D. M., \& Chaparro-Gutiérrez, J. J. (2019). Presence of Paragonimus species within secondary crustacean hosts in Bogotá, Colombia. Revista Colombiana de Ciencias Pecuarias, 32(2), 150-157. DOI:10.17533/udea.rccp.v32n2a08.

Pinheiro, M. A. A., Kriegler, N., de Souza, C. A., \& de Almeida Duarte, L. F. (2021). Feeding Habit and Lifestyle Influence the Baseline Micronuclei Frequency of Crab Species in Pristine Mangroves. Wetlands, 41(2), 1-14. DOI:10.1007/s13157-021-01427-4.

Quirós, J. A., \& Campos, N. H. (2010). Dinámica espacial de crustáceos decápodos asociados a céspedes algales en el departamento de Córdoba, Caribe Colombiano. Acta biológica. 15(3), 91-105. https://www.redalyc.org/articulo. oa?id $=319027886007$

Quirós, J., Dueñas, P., \& Campos, N. H. (2012). Crustáceos decápodos asociados a ensamblajes macroalgales en el litoral rocoso de Córdoba, Caribe colombiano. Revista $M V Z$
Córdoba, 17(1), 2834-2845. DOI:10.21897/rmvz.251.

Rangel-Buitrago, N. G. \& Posada-Posada, B. O. (2005). Geomorfología y procesos erosivos en la costa norte del departamento de Córdoba, Caribe colombiano (sector Paso Nuevo-Cristo Rey). Boletín de Investigaciones marinas y Costeras, 34(1), 101-119. DOI:10.25268/bimc.invemar.2005.34.0.236

Rivera-Pérez, J. M., Orrego-Meza, J. G., Escobar-Lasso, S., Zuluaga-Isaza, J. C., Londoño-Quiceno, C., \& Caicedo-Martínez, L. S. (2020). Unexpected case of cancrivory in a lotic tropical ecosystem: First record of predation of Strengeriana maniformis by the frog Rheobates palmatus. Food Webs, 25, e00163. DOI:10.1016/j.fooweb.2020.e00163.

Rodríguez, B. D. (1982a). Los cangrejos de la familia Portunidae (Decapoda: Brachyura) del Caribe colombiano. Boletín de Investigaciones Marinas $\&$ Costeras, 12(1), 137-184. DOI:10.25268/bimc.invemar.1982.12.0.501.

Rodríguez, G. (1980). Los crustáceos decápodos de Venezuela. Instituto Venezolano de Investigaciones Científicas.

Rodríguez, G. (1982b). Les crabes d'eau douce d'Amerique: Famille des Pseudothelphusidae. IRD Editions.

Rodríguez, G., \& Díaz., H. (1981). New species of freswater crabs from the Andes (Crustacea: Decapoda: Pseudothelphusidae). Senckenbergiana Biológica, 61(1), 305312.

Sánchez, H., \& Campos, N. H. (1978). Los Cangrejos ermitaños (Crustacea, Anomura, Paguridae) de la costa norte colombiana. Boletín de Investigaciones Marinas y Costeras 10(1), 15-62. DOI:10.25268/bimc.invemar.1978.10.0.503

Segadilha, J. L., \& Rodrigues, T. G. A. (2020). The crustacean collection at the National Institute of Mata Atlântica (INMA), former Professor Mello Leitão Biology Museum (MBML). Nauplius, 28, e2020046. Epub November 30, 2020. DOI:10.1590/2358-2936e2020046

Shih, H. T., Ng, P. K., Davie, P. J., Schubart, C. D., Türkay, M., Naderloo, R., Jones, D. \& Liu, M. Y. (2016). Systematics of the family Ocypodidae Rafinesque, 1815 (Crustacea: Brachyura), based on phylogenetic relationships, with a reorganization of subfamily rankings and a review of the taxonomic status of Uca Leach, 1814, sensu lato and its subgenera. Raffles Bulletin of Zoology, 64 (1), 139175. http://zoobank.org/References/ 80EBB258-0F6A-4FD6-9886-8AFE317C25F6

SIB Colombia. (2021, abril 11). Biodiversidad en Cifras, Sistema de Información sobre Biodiversidad de Colombia. https://cifras.biodiversidad.co/

Simmons, J. E., \& Muñoz-Saba, Y. (Eds.). (2005). Cuidado, manejo y conservación de las colecciones biológicas. Universidad Nacional de Colombia. Conservación Internacional. http://www.ibiologia.unam.mx/pdf/ directorio/c/cervantes/clases/sistem/Cuidado_Manejo_ y_Conservacion_de_las_Colecciones_Biologicas.pdf

Tobasura, I. A. (2008). Huella ecológica y biocapacidad: indicadores biofísicos para la gestión ambiental. El caso de Manizales, Colombia. Revista Luna Azul (On Line), 26(1), 120-136. https://revistasojs.ucaldas.edu.co/ index.php/lunazul/article/view/1098

Von Prahl, H., \& Manjarrés, G. (1984). Cangrejos Gecarcinidos (Crustacea; Gecarcinidae) de Colombia. Caldasia, 
14(66), 149-168. https://www.jstor.org/stable/23641468 Wang, Q., Wang, J., Wu, Q., Xu, X., Wang, P., \& Wang, Z. (2021). Insights into the evolution of Brachyura (Crustacea: Decapoda) from mitochondrial sequences and gene order rearrangements. International Journal of Biological Macromolecules, 170, 717-727. DOI:10.1016/j.ijbiomac.2020.12.210

Wolfe, J. M., Luque, J., \& Bracken-Grissom, H. D. (2021). How to become a crab: Phenotypic constraints on a recurring body plan. BioEssays. Problems and paradigms, 43(5), e2100020. DOI:10.1002/bies.202100020

Yang, C., Wenger, S. J., Rugenski, A. T., Wehrtmann, I. S., Connelly, S., \& Freeman, M. C. (2020). Neotropical freshwater crabs (Decapoda: Pseudothelphusidae) increase rates of leaf breakdown in a neotropical headwater stream. Nauplius, 28, e2020020. Epub June 15, 2020. DOI:10.1590/2358-2936e2020020 